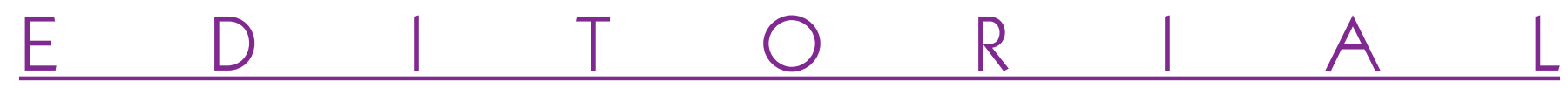

\title{
Breathing, dentofacial orthopedics, and health: the endorsement of licensing authorities
}

Jo doubt is to examine, to take ideas apart and put them together again, as you might with a watch works, without any preconceived notions and without haste.. Bernard Emile-Auguste CHARTIER, known as ALAIN, Propos.

n medicine, the development of ideas occasionally accelerates. Underlying reasoning is elaborated, profounder research is undertaken, and answers are found that more fully illuminate the pertinence of the formulas that suggested the original hypotheses.

In this way an obligatory connection has been made attesting to the perfect adequacy of the original inductive scientific procedure in the framework of evidence based dentofacial orthopedics.

The decree of March 301998 added to the official nomenclature treatment for respiratory insufficiency by rapid palatal expansion. This assumption, a tacit recognition of the medical benefits bestowed by dentofacial orthopedic treatment, would seem to be the first step in a laudable extension of the range of care that we deliver.

Another element of our orthopedic treatment arsenal has since received the endorsement of the High Health Authority (HHA), which has given its approbation to the therapeutic efficacy of orthopedic devices designed to advance the mandible in certain clinical situations, in the reduction, for example, of day time somnolence and correction of night time apneas and hypopneas. The HHA has stressed the public health importance of these maladies because of the high prevalence of sleep obstruction apnea and hypnopnea syndrome (SOAHS) and the need for its treatment in patients whose insurance will not pay for positive pressure ventilation devices or who are not able to tolerate them. With these new evaluations, the mandible has at last assumed an importance greater that its role as mastication's hammer. Its function in breathing and as the essential anchor for the various soft tissue structures that take part in the mechanism of breathing has been officially recognized.

Certainly, the deleterious effect SOAHS has on the quality of life and the possibilities of its contributing to cardio-vascular as well as respiratory complications have focused the attention of the 
medical world on the way people breathe. Still this malfunction should not become the leafy tree that obstructs our view of the thick forest of nasal disorders that lies behind it.

The optimization of passage through the upper airway (UA) is one of the major objectives of dentofacial orthopedics and the diagnosis of sleep obstruction problems is a key element in this enterprise.

By orthopedic treatment designed to improve the status of an oronasal complex that would be a candidate for eventual surgical suppression of anatomic obstacles and a course of medical treatment for respiratory allergies, orthodontists can contribute to the re-establishment of nasal breathing from early infancy, thus offering the child improved chances for satisfactory facial morphogenesis and providing the future adult with effective prevention of sleep obstruction disorders.

Orthodontists who conduct their practices in conformity with the principles of evi- dence based medicine will not limit the treatment choices they offer to patients only to those they are accustomed to using. They understand that just because they obtain a successful treatment result that the technique they employed was not necessarily the one best adapted to the original condition or that it provided the patient with the totality of the known possibilities known for correction of the patient's condition, especially in terms of the optimization of nasal breathing at rest and in cervicocephalic posture.

Limiting the treatment of breathing problems exclusively to the field of otolaryngology would restrict our area of competence and restrict us to the simple trade of moving teeth rather than exercising the wider scope of our competence as dentofacial orthopedists. What would be worse, that wouldn't just restrain our practices it would also deprive our patients of the opportunity to develop physically and psychosocially to the greatest possible extent. As orthopedists of the face, can we accept this as a reasonable situation?

Clearly, we must place the perspectives of our specialty well beyond our traditional objectives of obtaining a balanced occlusion in a harmonious face. Yes, we can... and, even more, we must, out of respect for our patients. 